\title{
Predictive control of wind farms based on lexicographic minimizers for power reserve maximization
}

\author{
Sara Siniscalchi-Minna, Fernando D. Bianchi, and Carlos Ocampo-Martinez, Senior Member, IEEE
}

\begin{abstract}
This paper presents a model predictive control (MPC) strategy aimed to regulate the total power delivered to the grid while maximizing the power reserve. Nowadays, the high participation of wind energy in the electricity generation requires that wind power plants (WPPs) also provide ancillary services. This fact implies that WPPs must be capable of temporally increasing the power generation to help, for instance, the primary-frequency control. To this end, WPPs work below the maximum generation capacity keeping some power reserves (difference between available and generated powers). The available power depends on the wind conditions that each turbine is facing but these conditions are also affected by the wakes produced by upstream turbines. In order to satisfy the aforementioned objectives, this work proposes to cast the MPC strategy as a multi-objective optimization problem solved using a lexicographic approach in order to consider the hierarchy of the control objectives. The performance of the control scheme is evaluated by simulations for the case of a WPP with three turbines taking into account the variation of wind speed faced by downstream turbines due to the wake effect.
\end{abstract}

\section{INTRODUCTION}

In the last decades the electrical power systems have experienced significant changes due to the increase in the installations of renewable energy sources (RES). Nowadays, the wind power capacity installed worldwide up to 2016 was $487 \mathrm{GW}$ with an electrical production become relevant in a scenario previously dominated by conventional power plants [1]. A downside of this high penetration of wind power (and other RES) in electrical networks is the reduction of the system inertia, making power systems more prone to frequency fluctuations and unstable behaviors [2]. As a consequence, some Transmission System Operators (TSO) are requiring wind power plants to contribute to the system stabilization as other conventional power plants [3]. However the power that a wind farm can deliver to the grid depends on the wind conditions that each turbine is facing. The wind conditions faced by a turbine are affected by the wakes produced by upstream turbines. Therefore, the power generated by each turbine depends not only on the meteorological conditions

S. Siniscalchi Minna is with Catalonia Institute for Energy Research, IREC, Jardins de le Dones de Negre, s/n Barcelona, Spain. ssiniscalchi@irec.cat

F. D. Bianchi is with CONICET and Instituto Balseiro, Bustillos 9500, S.C. Bariloche, Argentina. fernando.bianchi@ib.edu.ar

S. Siniscalchi Minna and C. Ocampo Martinez are with the Automatic Control Department, Universitat Politècnica de Catalunya, Institut de Robòtica i Informàtica Industrial (CSIC-UPC), Llorens i Artigas, 4-6, 08028 Barcelona, Spain. \{ssiniscalchi, cocampo\}eiri.upc . edu

This work has received funding from the European Union's Horizon 2020 research and innovation programme under the Marie Skodowska-Curie grant agreement No 675318 (INCITE) but also on the generation conditions of the upstream turbines [4].

Frequency fluctuations in an electrical grid are basically caused by temporal imbalances between consumed and generated powers. With the aim of participating in frequency control, wind farms must be able to deliver certain additional power in order to help in the power balance. Two main approaches have been proposed for this purpose: either to release the kinetic energy stored in the wind rotors or to operate wind turbines in de-loading mode. The first approach is mainly used to provide inertial frequency control, i.e., the first response aimed to stop the initial frequency drop. Kinetic energy is stored in the rotating masses of the turbines and released into the grid in case of a frequency event. Different strategies to inject kinetic energy have been proposed [5]-[7]. For instance, in [6] a scheduling of the generated power by each turbine is used as the set point for the speed controller in order to reduce the losses of kinetic energy during the normal operation mode. In [7], the stored kinetic energy is maximized by reducing the power generated by some wind turbines without reducing the total power. Deloading approaches aim mainly to contribute in the primary frequency control, which seeks to limit the fall and stabilize the frequency toward steady-state operating value. When the wind conditions and the power demanded by the TSO permit, wind farms work below their maximum power production capacity [8]. In this circumstance, the difference between the available and the generated powers results in a power reserve. This reserve capacity can be used to increase the generation in order to help in the primary frequency control when needed. In [9], several de-loading operations have been studied in order to provide active power control when the wake produced by the turbines is considered. The authors in [10] proposed methods to evaluate the impact of wakes on the power reserve.

In this paper, we propose a de-loading control strategy based on model predictive control (MPC). MPC has been successfully applied in wind farm control to address different objectives, such as minimizing the mechanical loads [11], [12], or coordinating energy storage system and wind farms [13]. In particular, MPC has been used to control the temporally power contributions of a particular wind turbine to help in the frequency control [14]. In the present paper, we propose an MPC strategy to regulate the total power delivered from a wind farm at a set-point given the TSO whereas the power reserve available for frequency support is maximized. The proposed wind farm control uses the information about the generated and available power in each 
turbine and solves a multi-objective optimization problem in order to coordinate the best power contribution for each wind turbine. The multi-objective optimization problem is solved using the lexicographic formulation introduced in [15]. In such way, the power reference tracking and the power reserve maximization are the objectives of a hierarchical optimization problem where the solution resulting considering only the first objective is used as constraint for an optimization problem considering only the second objective. As a preliminary result and to show the efectiveness of the approach, the proposed control strategy was evaluated in the case of a small wind farm with three-turbines in the same row, facing a uniform free-stream wind speed perpendicular to the rotor plane. The wake effect is estimated with the commonly used Jensen's models [16].

The remainder of this paper is organized as follows. The wind farm and wake modelling are presented in Section II. Section III describes the control problem and the proposed MPC strategy based on the lexicographic formulation. Section IV presents the results for a wind farm with three wind turbines and, finally, conclusions are drawn in Section V.

\section{WIND FARM MODELLING}

We consider the problem of regulating the total power delivered at the Point of Common Coupling (PCC) by a wind farm of $n_{t}$ turbines. The aerodynamic power generated by the $i$-th turbine is given by

$$
P_{g, i}=(\rho \pi / 2) R^{2} C_{p}\left(\lambda_{i}, \beta_{i}\right) v_{i}^{3},
$$

where $\rho$ is the air density, $R$ is the rotor radius, and $v_{i}$ the wind speed experienced by the turbine. The power coefficient $C_{p}$ depends on the pitch angle $\beta_{i}$ and the tip speed ratio $\lambda_{i}=\Omega_{i} R / v_{i}$, with $\Omega_{i}$ being the rotor speed.

The operation of a wind turbine can be divided into two regions as shown in Figure 1 (dashed line). In Region 1 (low wind speeds), wind turbines seek to maximize the energy capture, providing a power

$$
P_{\max , i}\left(v_{i}\right)=(\rho \pi / 2) R^{2} C_{p \max } v_{i}^{3} .
$$

In Region 2 (high wind speeds), the power is limited by the rated value $P_{\text {rated }}$. That is, the available power for a wind speed $v_{i}$ is

$$
P_{a v, i}= \begin{cases}P_{\text {max }, i}\left(v_{i}\right) & \text { if } v_{i} \leq v_{\text {rated }}, \\ P_{\text {rated }} & \text { if } v_{i}>v_{\text {rated }} .\end{cases}
$$

This available power is the maximum power that the $i$-th wind turbine can deliver when it is exposed to a wind speed $v_{i}$.

In the current context of high wind power participation in the electricity generation, wind turbines usually work in de-loading operation, injecting less power into the grid than the available power $P_{a v, i}$. In this circumstance, wind turbines work on the solid line in Figure 1, with $P_{r, i}$ being a set-point imposed by the farm controller. This fact allows keeping a certain power reserve

$$
P_{r e s, i}\left(v_{i}\right)=P_{a v, i}\left(v_{i}\right)-P_{g, i},
$$

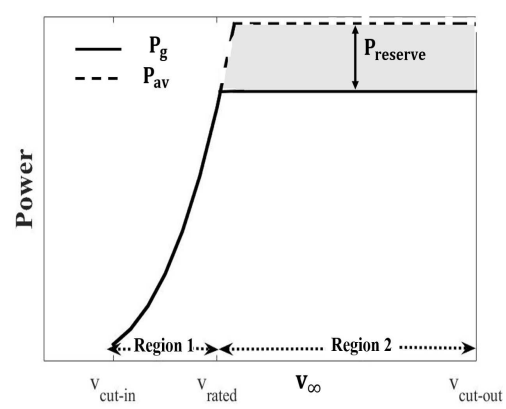

Fig. 1. Power-wind speed characteristics for each wind turbines. The dashed line corresponds to available power $P_{a v}$ and the solid line to the generated power actually delivered to the grid. The shaded area indicates the power reserve $P_{\text {res }}$ that can be used for frequency support.

which can be used for the provision of primary frequency support.

For the present analysis, it can be assumed that every wind turbine includes an internal power control strategy that ensures the power curve in Figure 1. In this circumstance, the dynamic behaviour from the power set-point to the generated power can be modelled as a first order system delivering a power

$$
\tilde{P}_{g, i}=\min \left\{P_{g, i}, P_{a v, i}\right\}
$$

where

$$
\dot{P}_{g, i}=\left(P_{r, i}-P_{g, i}\right) / \tau,
$$

being $\tau$ the time constant of the dynamic behaviour represented by means of a first-order model and $P_{r, i}$ the setpoint sent by the wind farm controller. In order to check the validity of this approximation, the simulations in Section IV have been performed with a more detailed model, including the nonlinear aerodynamic relationships.

The interaction between the wind rotor and the incoming free-stream wind speed $v_{\infty}$ perturbs the outflow field causing a phenomenon called wake effect. The disturbances affect the wind speed faced by the downstream turbines according to the farm layout and the direction of the wind speed. Modelling the wake effect is a complex task, but reasonable estimations can be obtained with relatively simple models such as the Jensen's model [16]. Figure 2 illustrates the wake effect for wind turbines in a row facing a uniform wind speed $v_{\infty}$ perpendicular to the rotor plane. Assuming a linear expansion of the wake having circular cross section $2 R_{w j}$ that increases proportionally to the distance between turbines, the downstream wind speed $v_{i}$ is computed as

$$
v_{i}=v_{\infty}\left(1-2 \sqrt{\sum_{j \in \mathbb{N}: x_{j}<x_{i}}\left(\left(1-\sqrt{1-C_{T, j}}\right) c_{j i}\right)^{2}}\right),
$$

where $\mathbb{N}=\left\{1, \ldots, n_{t}\right\}, C_{T, j}(\lambda, \beta)$ is the thrust coefficient, $c_{j i}=\left(2 R /\left(2 R+2 \alpha\left(y_{i}-y_{j}\right)\right)\right)^{2}, y_{j}$ and $y_{i}$ are the positions of the upstream and downstream turbines, respectively, and $\alpha$ the roughness coefficient that defines the slope of the wake when passing through a turbine.

Delivering a power profile $P_{d e m}$ at the PCC demanded by 


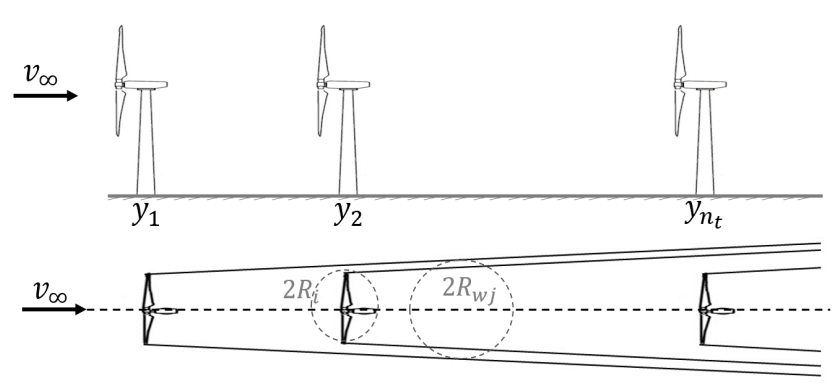

Fig. 2. Illustration of the wake effect for a set of wind turbines in a row.

the TSO can be achieved with different contributions from each wind turbine. This degree of freedom might be used to maximize the power reserve and thus increasing the wind farm capability for providing frequency support. However, as the available power $P_{a v, i}$ depends on the speed $v_{i}$ faced by the wind rotor, according to (3), the total available power in the wind farm, and also the total power reserve, will be affected by the contribution of each turbine. In Section III, we propose an MPC strategy to track a power profile at PCC while maximizing the power reserve.

\section{WIND FARM CONTROL FOR POWER RESERVE MAXIMIZATION}

\section{A. Control Problem Statement}

As mentioned in Section II, the control objectives considered in this paper are the following:

01: Ensure the tracking of a power demand profile $P_{\text {dem }}$.

O2: Maximize the total power reserve defined as

$$
P_{\text {res }}=\sum_{i=1}^{n_{t}} P_{\text {res }, i} \text {. }
$$

The former objective is basically a reference-tracking problem. The latter implies to coordinate the power contribution from each turbine taking into account the interaction caused by the wake effects in order to maximize the total power reserve. To satisfy these objectives, a centralized MPC strategy is proposed, which uses the $P_{g, i}$ and $P_{a v, i}$ sent by each turbine and the total power demand $P_{d e m}$ to produce the set-points $P_{r, i}$ for each turbine.

In order to ensure null steady-state error, the state-space model of the system is augmented with an integral action such as

$$
\dot{\xi}(t)=P_{\text {dem }}(t)-\sum_{i=1}^{n_{t}} P_{g, i} .
$$

Then, the system to be controlled is governed by

$$
\dot{\mathbf{x}}(t)=\mathbf{A x}(t)+\mathbf{B}_{1} \mathbf{P}_{r}(t)+\mathbf{B}_{2} P_{\text {dem }}(t),
$$

where $\mathbf{x}=\left[\mathbf{P}_{g}^{T}, \xi\right]^{T} \in \mathbb{R}^{n_{x}}$ is the state vector (with $n_{x}=n_{t}+1$ ), $\mathbf{P}_{g}=\left[P_{g, 1}, \ldots, P_{g, n_{t}}\right]^{T}$ is the vector of generated powers, $\mathbf{P}_{r}=$ $\left[P_{r, 1}, \ldots, P_{r, n_{u}}\right]^{T}$ denotes the vector of manipulated variables, and

$$
\mathbf{A}=\left[\begin{array}{cc}
-\frac{1}{\tau} \mathbf{I}_{n_{x}} & 0 \\
-\mathbf{1}_{n_{x}} & 0
\end{array}\right], \quad \begin{aligned}
& \mathbf{B}_{1}=[1 / \tau, \ldots, 1 / \tau, 0]^{T}, \\
& \mathbf{B}_{2}=[0, \ldots, 0,1]^{T},
\end{aligned}
$$

are the system matrices dependent from the time constant $\tau$ in the simplified wind turbine model (2). In (5), I is the identity matrix and $\mathbf{1}$ is a column vector of unitary entries, both of suitable dimensions.

In order to implement the MPC strategy, system (4) is discretized for a sampling time $T_{S}$, resulting in the following state-space difference equations:

$$
\dot{\mathbf{x}}(k+1)=\mathbf{A}_{d} \mathbf{x}(k)+\mathbf{B}_{d_{1}} \mathbf{P}_{r}(k)+\mathbf{B}_{d_{2}} P_{d e m}(k),
$$

with $k \in \mathbb{Z}_{\geq 0}$ the discrete-time instant and $\mathbf{A}_{d}, \mathbf{B}_{d_{1}}$ and $\mathbf{B}_{d_{2}}$ the discrete-time versions of matrices in (4). System states and manipulated variables are constrained because of physical and/or desired operational limits. These constraints are defined in a polytopic fashion as

$$
\begin{aligned}
& \mathbb{X}=\left\{\mathbf{x} \in \mathbb{R}^{n_{x}} \mid \mathbf{x}(k) \in[\underline{\mathbf{x}}, \overline{\mathbf{x}}], \forall k\right\}, \\
& \mathbb{U}=\left\{\mathbf{P}_{r} \in \mathbb{R}^{n_{u}} \mid \mathbf{P}_{r}(k) \in\left[\underline{\mathbf{P}}_{r}, \overline{\mathbf{P}}_{r}\right], \forall k\right\},
\end{aligned}
$$

where $\underline{\mathbf{x}} \in \mathbb{R}^{n_{x}}$ and $\overline{\mathbf{x}} \in \mathbb{R}^{n_{x}}$ denote the vectors of minimum and maximum admissible values of system states, respectively, while $\underline{\mathbf{P}}_{r} \in \mathbb{R}^{n_{u}}$ and $\overline{\mathbf{P}}_{r} \in \mathbb{R}^{n_{u}}$ denote the vectors of minimum and maximum admissible values of manipulated variables, respectively.

In order to design the MPC strategy for the considered system, let $\hat{\mathbf{u}}(k)$ be a sequence of feasible control inputs (manipulated variables) within a pre-establish prediction horizon denoted by $H_{p} \in \mathbb{Z}_{>0}$. Similarly, let $\hat{\mathbf{x}}(k)$ be the sequence of feasible system states when applying the control input sequence $\hat{\mathbf{u}}(k)$ to the system in (6). Finally, let $\hat{\mathbf{d}}(k)$ be the available forecasting of the power demands (disturbances). Hence,

$$
\begin{aligned}
& \hat{\mathbf{u}}(k) \triangleq\left\{\mathbf{P}_{r}(k \mid k), \ldots, \mathbf{P}_{r}\left(k+H_{p}-1 \mid k\right)\right\}, \\
& \hat{\mathbf{x}}(k) \triangleq\left\{\mathbf{x}(k+1 \mid k), \ldots, \mathbf{x}\left(k+H_{p} \mid k\right)\right\}, \\
& \hat{\mathbf{d}}(k) \triangleq\left\{P_{\text {dem }}(k \mid k), \ldots, P_{\text {dem }}\left(k+H_{p}-1 \mid k\right)\right\} .
\end{aligned}
$$

Thus, the MPC controller is designed by stating the following open-loop finite-horizon multi-objective optimization problem:

$$
\min _{\substack{\hat{\mathbf{u}}(k) \in \mathbb{U} \\ \text { s.t. }}} J(k) \triangleq \sum_{m=1}^{3} \sum_{i=0}^{H_{p}-1} J_{m}(k+i),
$$

$$
\begin{aligned}
& \mathbf{x}(k+j+1 \mid k)=\left[\begin{array}{lll}
\mathbf{A}_{d} & \mathbf{B}_{d_{1}} & \mathbf{B}_{d_{2}}
\end{array}\right]\left[\begin{array}{c}
\mathbf{x}(k+j \mid k) \\
\mathbf{P}_{r}(k+j \mid k) \\
P_{d e m}(k+j \mid k)
\end{array}\right], \\
& \mathbf{u}(k+j \mid k) \in \mathbb{U}, \\
& \mathbf{x}(k+j \mid k) \in \mathbb{X},
\end{aligned}
$$

for $j \in\left[0, H_{p}-1\right]$ in (9b) and (9c) whilst for $j \in\left[1, H_{p}\right]$ in $(9 d)$. Assuming that the optimization problem in (9) is 
feasible, its solution yields the optimal sequence

$$
\hat{\mathbf{u}}^{\star}(k) \triangleq\left\{\mathbf{P}_{r}^{\star}(k \mid k), \ldots, \mathbf{P}_{r}^{\star}\left(k+H_{p}-1 \mid k\right)\right\} .
$$

Therefore, following the receding horizon control philosophy, the controller applies to the system (6) the first control input from $\hat{\mathbf{u}}^{\star}(k)$, which corresponds with $\mathbf{P}_{r}^{\star}(k \mid k)$. Then, a new state vector is measured from (6) and the procedure is repeated for $k+1$.

Notice that the multi-objective cost function in (9a) is defined according to the stated objectives $\mathbf{O 1}$ and $\mathbf{O 2}$ as follows. Objective $\mathbf{O 1}$ is formulated as the minimization of the linear cost function

$$
J_{1}(k) \triangleq\|Q \mathbf{x}(k)\|_{1},
$$

being $Q$ a weighting matrix prioritizing the corresponding system state such as the minimization of the tracking error is achieved. Due to the cost convenience of wind power generation, this minimization has the highest priority in order to supply the TSO power demand in the best possible way.

On the other hand, it is also required the wind farm to contribute with ancillary services like primary frequency support. This feature can be achieved by releasing the power reserve into the grid when the available power is higher than the demand. Hence, maximizing the cost function

$$
J_{3}(k) \triangleq\left\|\mathbf{P}_{a v}-\mathbf{P}_{g}\right\|_{1},
$$

the control strategy may ensure the capability of the system for increasing the power generation when needed.

As a complementary action, the cost function

$$
J_{2}(k) \triangleq\left\|R \Delta \mathbf{P}_{r}(k)\right\|_{1},
$$

with $\Delta \mathbf{P}_{r}(k) \triangleq \mathbf{P}_{r}(k)-\mathbf{P}_{r}(k-1)$ and $R$ a weighting term of suitable dimensions, aims at smoothing the variation of the manipulated inputs and then avoiding undesired peaks in the output power signal.

\section{B. Proposed Approach}

Given the structure of the problem in (9) and the nature of cost functions in (10), (11) and (12), this paper proposes to solve the multi-objective optimization problem by using the sequential solution method of lexicographic minimizers [15], [17]. Here, the lexicographic minimization is applied to both define and ensure the hierarchy of the control objectives. Thereby, the lexicographic solution is a special type of Pareto-optimal solution that takes into account the order of the objectives. Consider a multi-objective optimization problem

$$
\min _{q \in \mathbb{Q}}\left[f_{1}(q), \cdots, f_{r}(q)\right],
$$

being $q \in \mathbb{Q} \subseteq \mathbb{R}^{p}$ the vector of the optimization variables and $f_{l}, l=1, \ldots, r$, scalar functions of $q$. Hence, the way of solving (13) follows the procedure shown in Algorithm 1. Notice that functions $f$ are arranged according to their priority from the most important $f_{1}$ up to the least important $f_{r}$.

Hence, regarding the combined problem of power reference tracking plus power reserve maximization addressed in

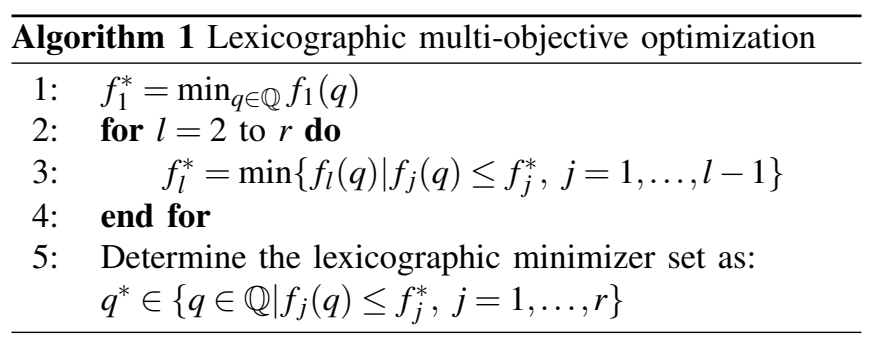

this paper, the solution of (9) is obtained by considering a first optimization problem taking into account cost functions (10) and (12), that is, an optimization problem composed of two linear cost functions properly prioritized such as the reference tracking was more important than the smoothness of the control input (by means of $Q$ and $R$ in (10) and (12), respectively). This optimization problem is therefore stated as

$$
\begin{array}{cl}
\min _{\hat{\mathbf{u}}(k) \in \mathbb{U}} & \sum_{i=0}^{H_{p}-1} J_{1}(k+i)+J_{2}(k+i) \\
\text { s.t. } & (9 \mathrm{~b}),(9 \mathrm{c}) \text { and }(9 \mathrm{~d}),
\end{array}
$$

from which an optimal sequence $\hat{\mathbf{u}}_{1}^{\star}(k)=\hat{\mathbf{u}}^{\star}(k)$ is obtained and used to define an extra constraint for a second optimization problem focused on the maximization of the power reserve, i.e.,

$$
\begin{array}{cl}
\max _{\hat{\mathbf{u}}(k) \in \mathbb{U}} & \sum_{i=0}^{H_{p}-1} J_{3}(k+i) \\
\text { s.t. } & (9 \mathrm{~b}),(9 \mathrm{c}) \text { and }(9 \mathrm{~d}), \\
& \sum_{i=0}^{H_{p}-1} J_{1}(k+i)+J_{2}(k+i) \leq \hat{\mathbf{u}}_{1}^{\star}(k)+\varepsilon
\end{array}
$$

where $\varepsilon>0$ is a small tolerance in order to avoid numerical problems and the infeasibility of the optimization problem in (15). Finally, the resultant optimal sequence $\hat{\mathbf{u}}_{2}^{\star}(k)$ from the solution of (15) corresponds with the definitive sequence from which the first element $\hat{\mathbf{u}}_{2}^{\star}(k \mid k)=P_{r}^{\star}(k \mid k)$ will be taken and applied to the system.

\section{CAse Study}

The proposed control strategy was evaluated in the case of a wind farm with three turbines in a row $\left(n_{t}=3\right)$. The turbines correspond to the $5 \mathrm{MW}$ benchmark introduced in [18]. For the simulations, the wind turbines were described by a nonlinear two-mass model including also the pitch actuator and a power control as proposed in [19]. The turbines were considered aligned with the free-stream wind speed direction and the distance between turbines was $882 \mathrm{~m}$ (seven rotor diameters).

The MPC strategy was implemented with a sampling time $T_{s}=0.01 \mathrm{~s}$ and a prediction horizon $H_{p}=4$, which have been set to be relatively small since the turbines are required to regulate power on millisecond scale. The time constant $\tau$ in the simplified model (2) was set at $0.08 \mathrm{~s}$, which was obtained from the step response of the more detailed wind turbine model. The parameters in the cost functions 

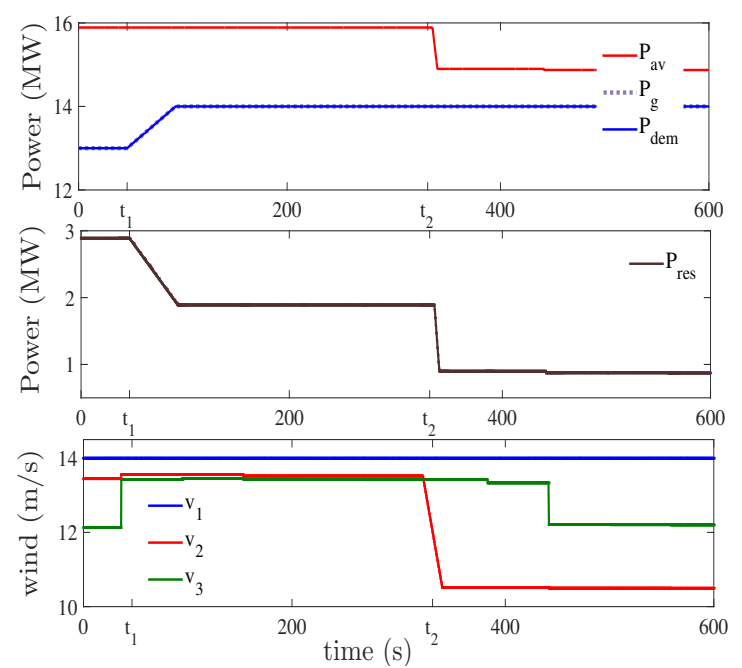

Fig. 3. System response for scenario 1. Top plot: generated, available and demanded total power. Middle plot: total power reserve. Bottom plot: wind speed faced by each turbine.

(10) and (11) were set as $Q=\operatorname{diag}\left[\begin{array}{llll}0 & 0 & 0 & 26.6\end{array}\right]$ and $R=10^{-5}$. Simulations have been run using YALMIP [20] and CPLEX under MATLAB ${ }^{\circledR}$ in a PC with an Intel i7 processor and 8 Gb RAM.

In order to evaluate how the controller coordinates the power contribution of each turbine, two scenarios were considered. The first scenario analyzes changes in the power demand set-point. The second evaluates the system response under a change of the free-stream wind speed. In both cases, the free-stream wind speed was set in order to ensure that the available power was higher than the one demanded by the TSO.

\section{A. Scenario 1: Changes in power demand set-point and wind speed $v_{2}$}

This scenario analyzes the system behavior when the power set-point $P_{d e m}$ increases from 13 to $14 \mathrm{MW}$ (at $t_{1}=100 \mathrm{~s}$ ) and the wind speed $v_{2}$ falls to $10.5 \mathrm{~m} / \mathrm{s}$ (at $t_{2}=360 \mathrm{~s}$ ). The initial free-stream wind speed was $12 \mathrm{~m} / \mathrm{s}$. The system response with the proposed control strategy is shown in Figure 3. In the top plot, it can be observed the power demand set-point $P_{d e m}$ (blue line), the total generated power $P_{g}$ (dashed line) and the total available power $P_{a v}$ (red line). The middle plot shows the total power reserve and the bottom plot the wind speeds $v_{1}, v_{2}$ and $v_{3}$ faced by each wind turbine, respectively. The generated and available powers for each wind turbine is displayed in Figure 4, with solid and dashed lines, respectively.

As shown in Figure 3, the control is able to achieve a proper tracking in spite of the disturbances, delivering to the grid the power demanded by the TSO. This tracking is achieved by increasing the power contribution of each turbine (Figure 4), which reduces the total power reserve and slightly affects the wind speed faced by Turbines 2 and 3 due to the wake effects. The subsequent reduction of the wind speed $v_{2}$ for $t<t_{2}$ causes a significant decrease
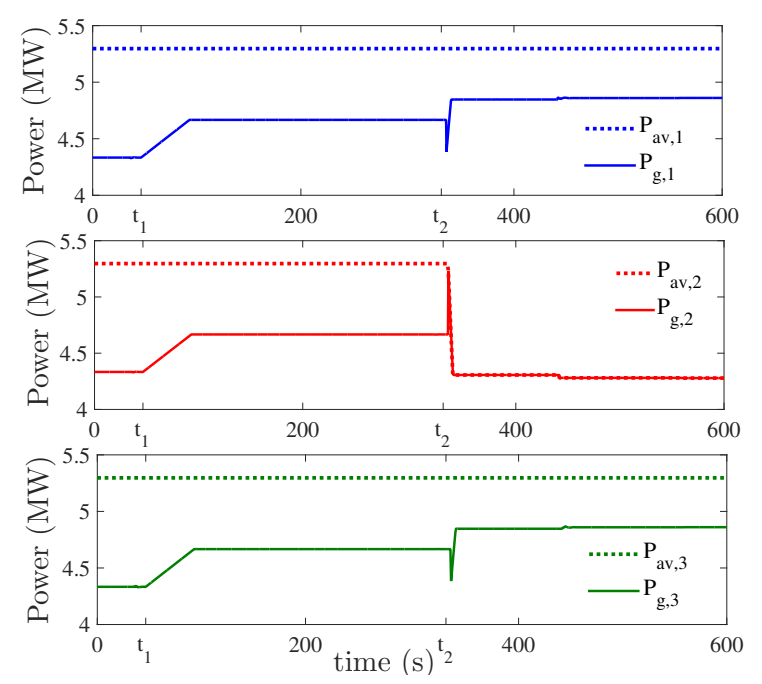

Fig. 4. System response for scenario 1: generated and available powers for each turbine.

in the available power in Turbine 2. After $t_{2}$, Turbine 2 is forced to work at maximum generation. As a consequence, the controller re-distributes the power contribution of the remaining turbines increasing $P_{g, 1}$ and $P_{g, 3}$. Nevertheless, the total power demanded at the PCC is still satisfied. In Figure 4 , it can be seen that $v_{3}$ decreases once the wake disturbance caused by the increase $P_{g, 1}$ finally arrived at Turbine 3. However, this does not affect $P_{a v, 3}$ because the wind speed $v_{3}$ is still sufficient to operate at region 2 (Figure 1).

\section{B. Scenario 2: Change in free-stream wind speed and in $v_{2}$}

The second scenario considers a change in the free-stream wind speed $v_{\infty}=v_{1}$ from 14 to $13 \mathrm{~m} / \mathrm{s}$ at $t_{3}=150 \mathrm{~s}$ followed by an additional reduction in $v_{2}$ at $t=360 \mathrm{~s}$. These changes can be observed in the bottom plot in Figure 5. It can be also seen in Figure 5 that the control is able to manage this situation keeping the total power delivered to the grid, but a significant reduction in the available power, and consequently in the reserve, occurs after $t=t_{2}$.

Figure 6 shows the generated and available powers corresponding to each turbine. The first change in the freestream wind speed does not have a significant effect on generated and available powers as the wind speeds faced by each turbines ensure the operation in region 2 (Figure 1). On the other hand, the subsequent reduction of $v_{2}$ forces Turbine 2 to enter in region 1 and delivered all its available power $\left(P_{g, 2}=P_{a v, 2}\right)$. The lower contribution from Turbine 2 must be compensated with increments in the power supplied for the remaining turbines. In Figure 6, it can be observed that once the increase in $P_{g, 1}$ starts to affect the wind speed $v_{3}$, $P_{g, 3}$ also reaches the available power $P_{a v, 3}$.

\section{CONCLUSiOnS}

This paper has proposed an MPC strategy for the regulation of the power generated by a wind farm. Currently, WPPs must contribute to the grid support as other conventional 

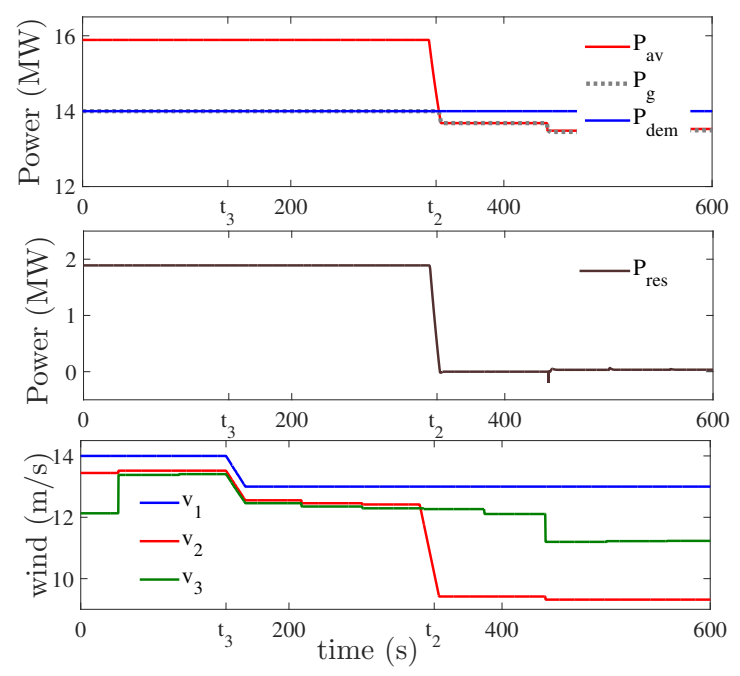

Fig. 5. System response for scenario 2. Top plot: generated, available and demanded total power. Middle plot: total power reserve. Bottom plot: wind speed faced by each turbine.

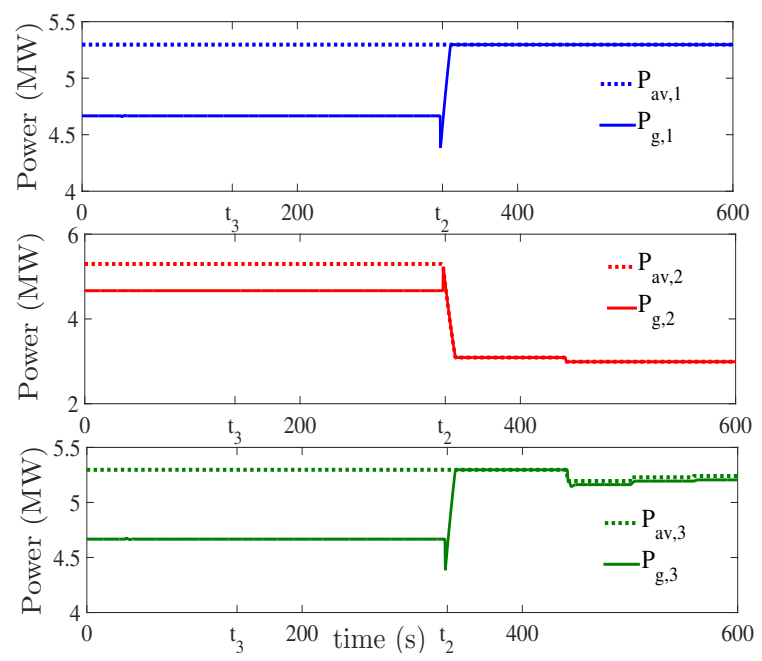

Fig. 6. System response for scenario 2: generated and available powers for each turbine.

power sources. To this end, WPPs must keep some power reserve that allows them to increase the power generation and thus help, for instance, the primary frequency control. Unlike conventional power sources, to keep a certain power reserve in a WPP requires the coordination of many small generators (turbines) that are exposed to different conditions (wind and generated power) coupled each others due to the wake effects. Here, we have proposed a multi-objective MPC scheme aimed to regulate the total power generated and coordinate the contribution of each turbine to maximize the power reserve. The control seeks to exploit the degree-offreedom in the distribution of the power contribution of each turbine to obtain the total demanded power and maximize the total power reserve. This strategy has been evaluated in a simple array of three wind turbines showing promising results. As future work, motivated by the fact that usually within a wind farm there are tens of turbines, it is proposed to test the MPC strategy for more complex wind farm layout.

\section{REFERENCES}

[1] WWEA, "WWEA half-year report: worldwind capacity reached 456 GW," Oct. 2016.

[2] ENTSO-E, "Future system inertia," Apr. 2014.

[3] S. Boersma, B. Doekemeijer, P. Gebraad, P. Fleming, J. Annoni, A. Scholbrock, J. Frederik, and J. van Wingerden, "A tutorial on control-oriented modeling and control of wind farms," in Proc. of American Control Conference (ACC), 2017, pp. 1-18.

[4] S. Frandsen, R. Barthelmie, S. Pryor, O. Rathmann, S. Larsen, J. Højstrup, and M. Thøgersen, "Analytical modelling of wind speed deficit in large offshore wind farms," Wind energy, vol. 9, no. 1-2, pp. 39-53, 2006.

[5] A. Zertek, G. Verbic, and M. Pantos, "A novel strategy for variablespeed wind turbines' participation in primary frequency control," IEEE Transactions on Sustainable Energy, vol. 3, no. 4, pp. 791-799, 2012.

[6] A. De Paola, D. Angeli, and G. Strbac, "Scheduling of wind farms for optimal frequency response and energy recovery," IEEE Transactions on Control Systems Technology, vol. 24, no. 5, pp. 1764-1778, 2016.

[7] A. S. Ahmadyar and G. Verbič, "Coordinated operation strategy of wind farms for frequency control by exploring wake interaction," IEEE Transactions on Sustainable Energy, vol. 8, no. 1, pp. 230-238, 2017.

[8] R. G. De Almeida and J. P. Lopes, "Participation of doubly fed induction wind generators in system frequency regulation," IEEE Transactions on Power Systems, vol. 22, no. 3, pp. 944-950, 2007.

[9] P. Fleming, J. Aho, P. Gebraad, L. Pao, and Y. Zhang, "Computational fluid dynamics simulation study of active power control in wind plants," in American Control Conference (ACC), 2016, 2016, pp. 1413-1420.

[10] T. G. Bozkurt, G. Giebel, N. K. Poulsen, and M. Mirzaei, "Wind speed estimation and parametrization of wake models for downregulated offshore wind farms within the scope of posspow project," in Journal of Physics: Conference Series, vol. 524, no. 1. IOP Publishing, 2014, p. 012156.

[11] V. Spudic, M. Jelavic, M. Baotic, and N. Peric, "Hierarchical wind farm control for power/load optimization," The science of making torque from wind (Torque2010), 2010.

[12] B. Biegel, D. Madjidian, V. Spudic, A. Rantzer, and J. Stoustrup, "Distributed low-complexity controller for wind power plant in derated operation," in Proc. IEEE International Conference on Control Applications (CCA), 2013, pp. 146-151.

[13] H. Zhao, Q. Wu, Q. Guo, H. Sun, and Y. Xue, "Optimal active power control of a wind farm equipped with energy storage system based on distributed model predictive control," IET Generation, Transmission \& Distribution, vol. 10, no. 3, pp. 669-677, 2016.

[14] P. F. Odgaard, T. G. Hovgaard, and R. Wiesniewski, "Model predictive control for wind turbine power boosting," in Proc. IEEE European Control Conference (ECC), 2016, pp. 1457-1462.

[15] K. Miettinen, Nonlinear multiobjective optimization. Springer Science \& Business Media, 2012, vol. 12.

[16] N. O. Jensen, "A note on wind generator interaction," Roskilde, Denmark, Tech. Rep., 1983.

[17] C. Ocampo-Martinez, A. Ingimundarson, V. Puig, and J. Quevedo, "Objective Prioritization Using Lexicographic Minimizers for MPC of Sewer Networks," IEEE Transactions on Control Systems Technology, vol. 16, no. 1, pp. 113-121, 2008.

[18] J. Jonkman, S. Butterfield, W. Musial, and G. Scott, "Definition of a 5mw reference wind turbine for offshore system development," National Renewable Energy Laboratory, Golden, CO, Technical Report No. NREL/TP-500-38060, 2009.

[19] J. D. Grunnet, M. Soltani, T. Knudsen, M. N. Kragelund, and T. Bak, "Aeolus toolbox for dynamics wind farm model, simulation and control," in The European Wind Energy Conference \& Exhibition, EWEC, 2010.

[20] J. Lofberg, "Yalmip: A toolbox for modeling and optimization in MATLAB," in Proc. IEEE International Symposium on Computer Aided Control Systems Design, 2005, pp. 284-289. 